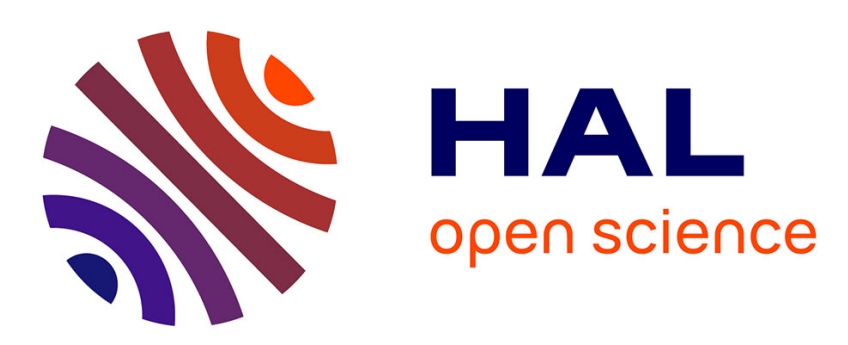

\title{
A knowledge database of qualified digitizing systems for the selection of the best system according to the application
}

\author{
C Mehdi-Souzani, Yann Quinsat, C Lartigue, P Bourdet
}

\section{- To cite this version:}

C Mehdi-Souzani, Yann Quinsat, C Lartigue, P Bourdet. A knowledge database of qualified digitizing systems for the selection of the best system according to the application. CIRP Journal of Manufacturing Science and Technology, 2016. hal-01255312

\section{HAL Id: hal-01255312 https://hal.science/hal-01255312}

Submitted on 22 Jan 2016

HAL is a multi-disciplinary open access archive for the deposit and dissemination of scientific research documents, whether they are published or not. The documents may come from teaching and research institutions in France or abroad, or from public or private research centers.
L'archive ouverte pluridisciplinaire HAL, est destinée au dépôt et à la diffusion de documents scientifiques de niveau recherche, publiés ou non, émanant des établissements d'enseignement et de recherche français ou étrangers, des laboratoires publics ou privés. 
A knowledge database of qualified digitizing systems for the selection of the best system according to the application

\author{
C. Mehdi-Souzani, Y. Quinsat, C. Lartigue, P. Bourdet
}

LURPA, ENS Cachan, Univ. Paris-Sud, Université Paris 13, Sorbonne Paris Cité, Université Paris-Saclay, 94235 Cachan, France

Abstract: Digitizing systems are widely used in industry for applications such as Reverse Engineering or inspection. Given the diversity of solutions, the selection of the most appropriate systems for an application has become a challenging task. To be efficient, system selection must rely on a knowledge base of the digitizing system performance with regard to the given application. Within this context, this paper aims at presenting how a knowledge database of qualified digitizing systems can be established accor ding to ability and quality criteria. The best system is afterwards obtained by optimizing a cost function built as the weighting sum of the criteria, weighting depending on the considered application.

Keywords: Knowledge based system, Reverse Engineering, Inspection, non-contact sensor, decision process

\title{
1. Introduction
}

Considering the large diversity of digitizing systems now proposed, the choice of the most appropriate system for a given application can be difficult. This has become a critical issue as applications are more and more diversified -Reverse Engineering, part inspection, rapid copying, dynamical balancing, and so on - and have different requirements. The selection is generally done by an expert in connection with the application requirements.

In the literature some studies address this issue. In [1], the authors proposed to select the most appropriate system for an application of old mechanismredesigning based on a decision tree. The choice relies on various criteria classified into categories: operability factors (whether completeness is required or not, whether palpation is authorized or not ...), and data on the object to be digitized (material, accessibility, accuracy, dimensions ...).To perform the selection, those criteria (or categories) are hierarchized as a decision tree. For applications of part inspection, Savio et al. |2] present a selection guide of the digitizing system type based on the object dimensions and the measuring uncertainty. These authors propose a classification of the types but the approach remains qualitative.Loriot [3] introduces several criteria, such as acquisition time, accuracy, material texture, ergonomics, and so on, to classify digitizing systems for duplication, visualization and analysis applications that belong to the computer graphics field. The aim of his study is to define a hierarchy of the applications for a given system. Barbero et al.[4] perform a comparative study of various digitizing systems based on the evaluation of different criteria: accuracy, density of points, completeness, etc. The study only focuses on system assessment and not on the selection of the most appropriate one in relation with an application.In [5], a measuring system selection is presented for an application of part inspection based on various factors such as sensor uncertainty, geometric attributes, material, surface finish and part flexibility.They propose to select the most suited sensor using a knowledge-based method. But, in practice, the knowledge is simply formalized as basic rules: a tactile probe is not suitable for flexible parts,the sensor uncertainty should satisfy the requirement of the tolerance being measured, etc. If such an approach seems interesting, the list of all the rules used is not clearly detailed. Moreover, the approach is only implemented for a laser scanner and a touch probe.For a specific application of crankshaft balancing, Zuquete et al. [6] propose to select the best-suited system by assessing optical digitizing systemsthanks toindicators. Those indicators - noise, trueness, accessibility, and measured area, are obtained through a specific protocol based on the measurement of simple 
artefacts.Audfrayet al.|7] extend this work by defining an assessment protocol applicable to a large range of digitizing systems and suggest storing information in databases. The first database is provided by the manufacturer's information and is used to select admissible systems according to criteria of ability. The second one, which results from the assessment protocol, is referred to as the qualified digitizing system database and gathers performance indicators (digitizing noise, trueness, acquisitiontime, etc.) that are used to elaborate a cost function. The best system is thus selected among the admissible systems as the one that minimizes the cost function built as the weighting sum of the indicators.

Methods proposed in the literature do not answer the general issue of finding the best digitizing system according to an application that could be RE, copying, part inspection, etc. They are most generally only dedicated to a given application, or if they consider various applications, they propose a classification of the systems but not a selection of the most appropriate system. Nevertheless, we can bring out some interesting key points. First, selection methods rely on criteria that can be classified into categories. Then, knowledge-based methods seem relevant to help in finding the most appropriate system, as for instance by criteria hierarchization as a decision tree [1]or by the use of simple rules [5].

The approach developed by Audfray et al. 17], applied first to part inspection, is interesting as the selection is made on the basis of non-subjective and evaluable performance indicators.It could be easily extended to other applications by linking the weightings with the considered application. This would require a change in the database structure so that it would include a database of applications. Within this context, this paper aims at presenting how a knowledge-based system can be used for the selection of the best digitizing system for a given application.As it will be described in section 3 , a digitizing system is a couple sensor/device. The originality of the approach is that a large diversity of triangular-based sensors (laser-plane, structured light, etc.) can be considered for a wide variety of applications (RE, part inspection, crankshaft balancing, etc.).

\section{Knowledge for the selection of the best digitizing system}

The idea is to design and develop a knowledge-based system that can be used systematically for the selection of the best digitizing system in relation with a given application. Therefore, the proposed approach inspired by the method proposed in [8] couples an efficient and systematic knowledge database with a decision system to support the optimal selection satisfying the application and the user constraints.The aim is the use and the communication of knowledge between different users; therefore, we consider knowledge within an objectivist perspective for which formal knowledge is preferred to tacit knowledge [9][10]. In this direction, the proposed knowledge-system consists of two databases.The first one concerns the digitizing systems and gathers qualified information issued from an assessment protocol. The originality is here that this database is built from objective information, as the protocol is the same for all digitizing systems. The second database is designed to embed and structure the expert's knowledge concerning the various applications requiring a digitizing system. The objectiveis to limit the intervention of the expert to this database construction, and not to the decision process.Then, the selection of the best system for a given application is performed through a decision process based on the assessment of performance functions. 


\section{Database of qualified digitizing systems}

\subsection{Database structure}

A digitizing system is defined by a sensor/devicecouple [7]. The sensors, or acquisition systems, are generally classified into two main categories: contact or non-contact sensors. The latter are most often optical sensors based on triangulation techniques, tomography or vision.

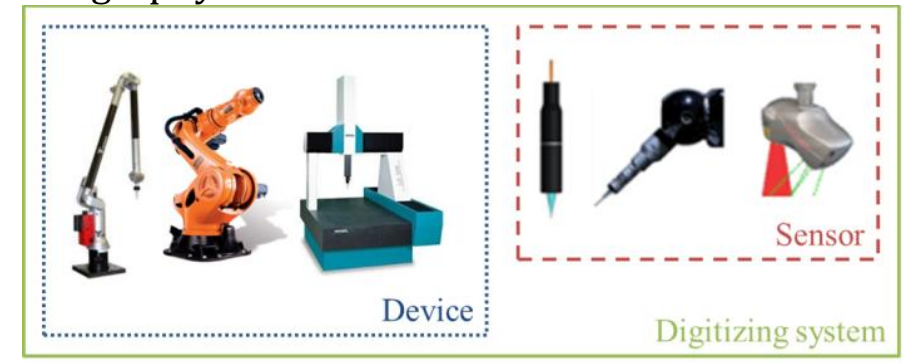

Figure 1. Digitizing systems

The device, or displacement system, allows the relative sensor/surface positioning throughout the whole digitizing process. The most classical devices include 3D positioning systems: CMM, machine tools, robots or articulated arms. Either sensors or devices possess intrinsic factors corresponding to manufacturer's data such as (without being exhaustive) sensor technology, field of view, resolution, device working space, acquisition speed, accuracy, etc. These characteristics are essential for the digitizing system selection, but the main difficulty is that they are not comparable. For instance, manufacturers give a value of accuracy but they generally do not specify how this value is obtained, and when they specify their protocol,the conditions under which it is obtained varyin function of the manufacturer. The selection of the best digitized system must lead to the choice of a sensor and a device according to non-subjective criteria. It is thus necessary to build objective indicators allowing the comparison between systems.

In the literature, some authors propose to classify the selection criteria according to categories. In [1] for instance, authors separate operability factors from factors related to the part to be measured. Classification according to quality and/or performance criteria is also addressed [5-7]. Within this context, we propose to define 3 main categories of indicators. Some of them are directly built from the intrinsic factors, whereas others result from a specific protocol.

- Ability indicators; they account for the aptitude of the system according to the measuring scale, the part properties (rigidity, color, material ...), the accessibility, and the measurement environment (off-line or on-line).

- Quality indicators; they account for the quality of the acquired data; the most common indicators are the digitizing noise, and the trueness.

- Operational indicators;they are generally associated to the acquisition performance,as for the completeness, the point density, the measured area, or the acquisition speed.

Quality and operational indicators result from an assessment protocol, independent of the user and the manufacturer, which will be detailed in next sections, whereas ability indicators are generally built from intrinsic factors (figure 2). 


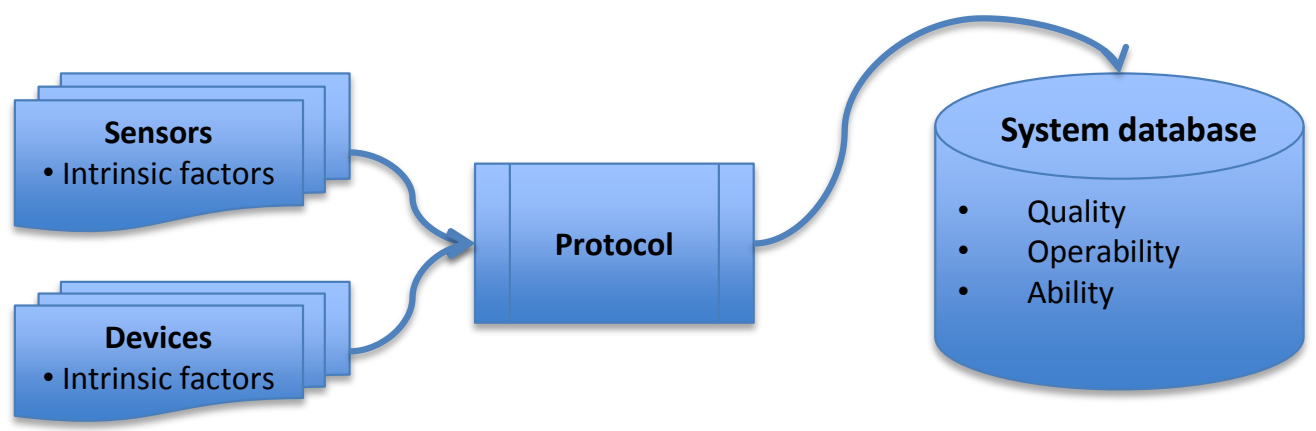

Figure 2. Definition of the qualified digitizing system database

\subsection{Assessment protocol for quality indicators}

Most studies proposed in the literature investigate quality of digitized data through quality indicators, generally evaluated by measurements of artefacts. The noise and the trueness are now commonly used to qualify a digitizing system [11-15].

The noise is evaluated by measuring a reference surface, whose theoretical model is well-known. More generally, the reference surface is a plane. Considering a set of $m$ points $x_{i}$ obtained after digitizing, and considering the ideal element $E$ corresponding to the expected surface, the noise is calculated using the following equation:

$$
\delta=k . \sigma=k \cdot \sqrt{\frac{1}{m} \sum_{1}^{m}\left(d_{i}-\bar{d}\right)^{2}}
$$

Where $d_{i}$ is the distance of each digitized point to the ideal element, $\bar{d}$ is the mean value of the distances, $\sigma$ is the standard deviation and $k$ is the coverage factor ( $k$ is generally set to 1). The artefact chosen is a plane with a certified low form deviation (Figure 3).The noise varies in function of the relative configuration sensor/part, i.e. in function of both the view angle and the digitizing distance [11-16].The evaluation of the noise $\delta$ is thus performed for several configurations.
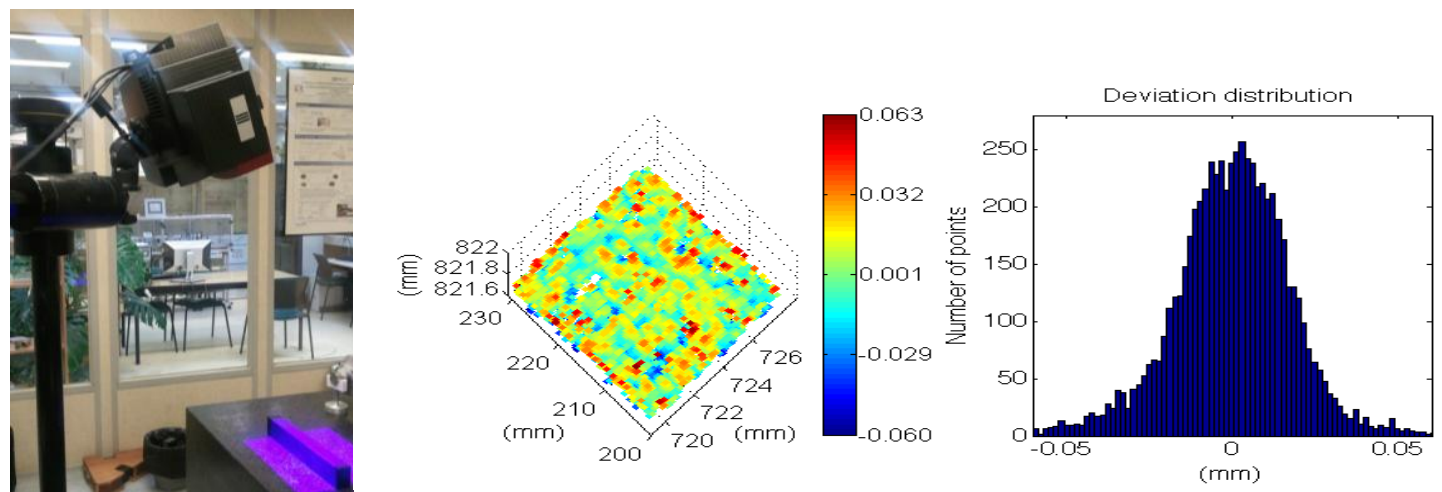

Figure 3. Evaluation of the digitizing noise

First, the digitizing distance is investigated. For this purpose, the digitizing distance D is defined according to the position of the object within the field of view (fov) as proposed in figure 4. The fov can be planar (case of laser-plane sensors for instance) or pyramidal (case of structured light sensors for instance) in function of the sensor technology. The distance $\mathrm{D}$ represents the distance between the emission point and the surface object. To study the influence of the digitizing distance only, the sensor is placed normally to the surface for various distances D according to the height of the fov. 


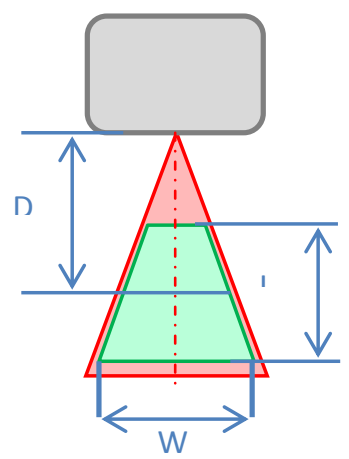

a- planar fov

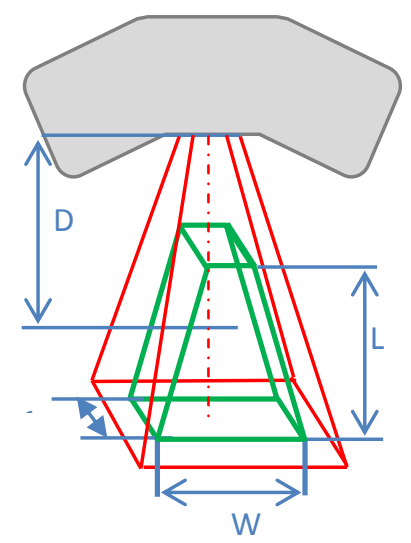

b- pyramidal fov
Laser area coverage

Digitizing area

$D$ : digitizing distance

$L$ : fov height

W: fov width

I : fov length

Figure 4. Definition of the field of view

This protocol is applied to various systemswith the aim of feeding the qualified database [7]. Some of these results are reported in table 1, in which 3 systems are more particularly studied. Table 1 represents an excerpt of the qualified digitizing system database, as it will be discussed in section 4. As an example, results obtained for a system composed by a laser-plane sensor mounted on a CMM (referred as to system 3) are displayed in figure 5. The noise increases with the digitizing distance, but remains less than $15 \mu \mathrm{m}$.
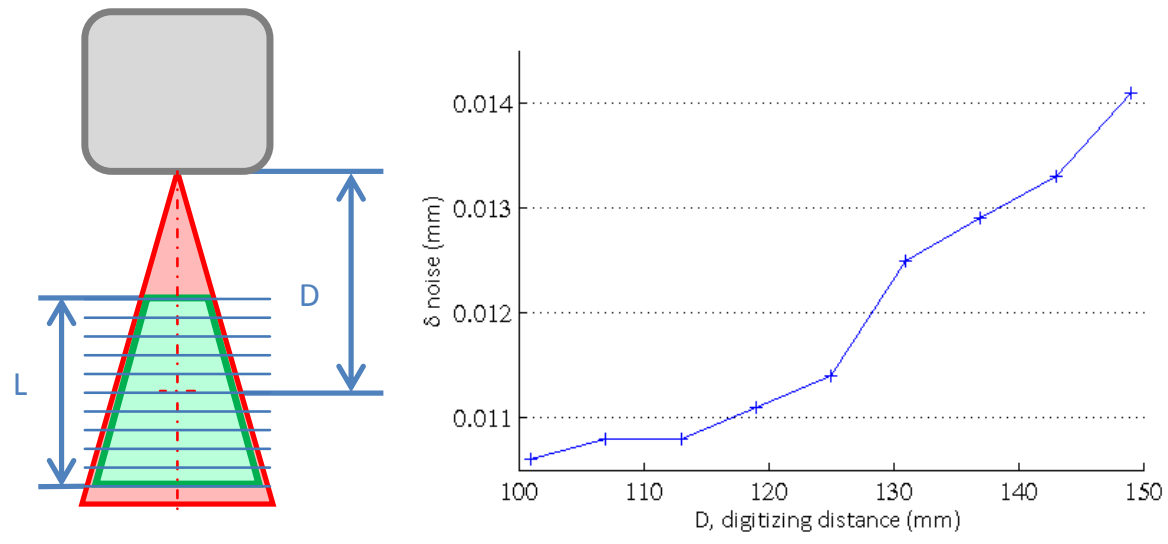

Figure 5. Evolution of the digitizing noise in function of the digitizing distance

The influence of the digitizing angles is also studied. For laser-plane sensors, two angles are defined, the in-plane angle $(\beta)$ and the out-off-plane angle $(\alpha)$ [14-16]. For structured light sensors, one angle can be defined between the optical axis of the projector and the normal vector to the surface. The influence of the in-plane angle $(\beta)$ is not investigated as it is generally less important than the influence of the out-off angle $(\alpha)$ [15]. Then, the influence of the out-off-plane angle $(\alpha)$ is investigated according to a set-up proposed in figure 6 ; the angle is modified thanks to a gauge stacking for which the height can be easily modified. Results reported in figure 6 for System 3, highlight a surprising behavior: the noise decreases with the view angle. Furthermore, the noise is maximum for $\alpha=10^{\circ}$. This is likely due to a glare phenomenon of the camera inherent to laser-plane technology. Except for this value, the digitizing noise remains less than $17 \mu \mathrm{m}$. 

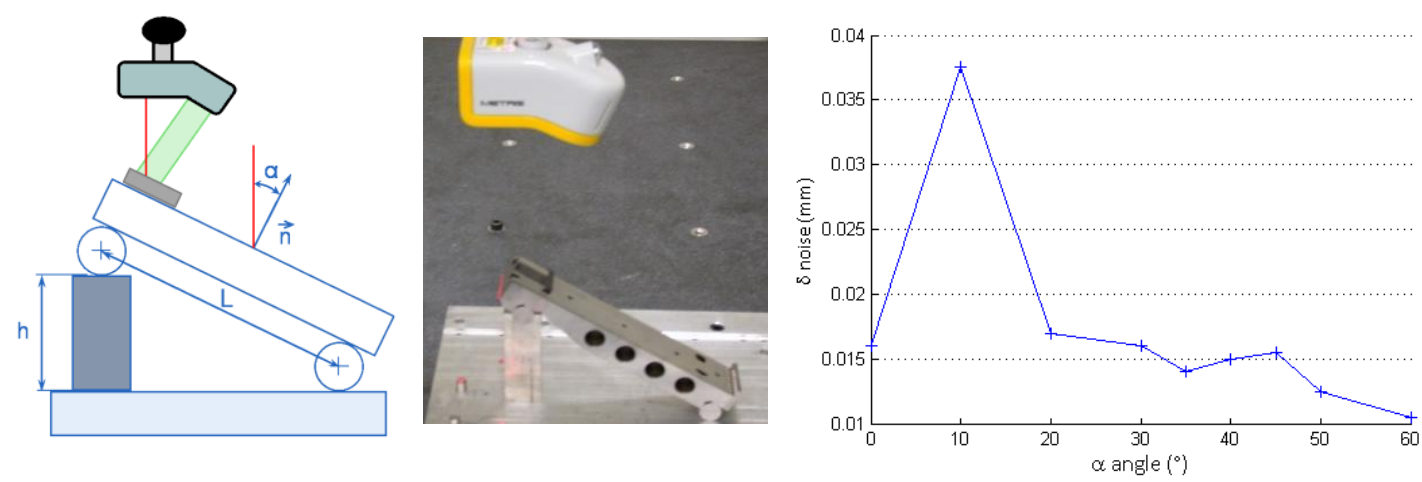

Figure 6. Evaluation of the digitizing noise

The trueness of a measurement is defined as the closeness of agreement between the average of an infinite number of replicate measured quantity values and a reference quantity value [17]. As the quantity value can be defined by a distance,the trueness is investigated by the measurement of a reference distance which can be the distance between two parallel planes defining a step height [6][7] or the distance between the 2 sphere centers of a ball-bar.Let us consider that the referencedistance $h_{0}$ is given by the distance between two parallel planes of a step (Figure 7). The digitizing of the step gives two point clouds, each one corresponding to a plane. A theoretical surface is associated to each point cloud according to the least-square criterion. Each plane $\left(P_{1}\right.$, resp. $\left.P_{2}\right)$ is thus characterized by a point $\left(A_{1}\right.$ resp. $\left.A_{2}\right)$, barycenter of each point cloud, and the normal vectorof each plane ( $\boldsymbol{n}_{1}$ resp. $\boldsymbol{n}_{2}$ for $P_{1}$, resp. $P_{2}$ )).
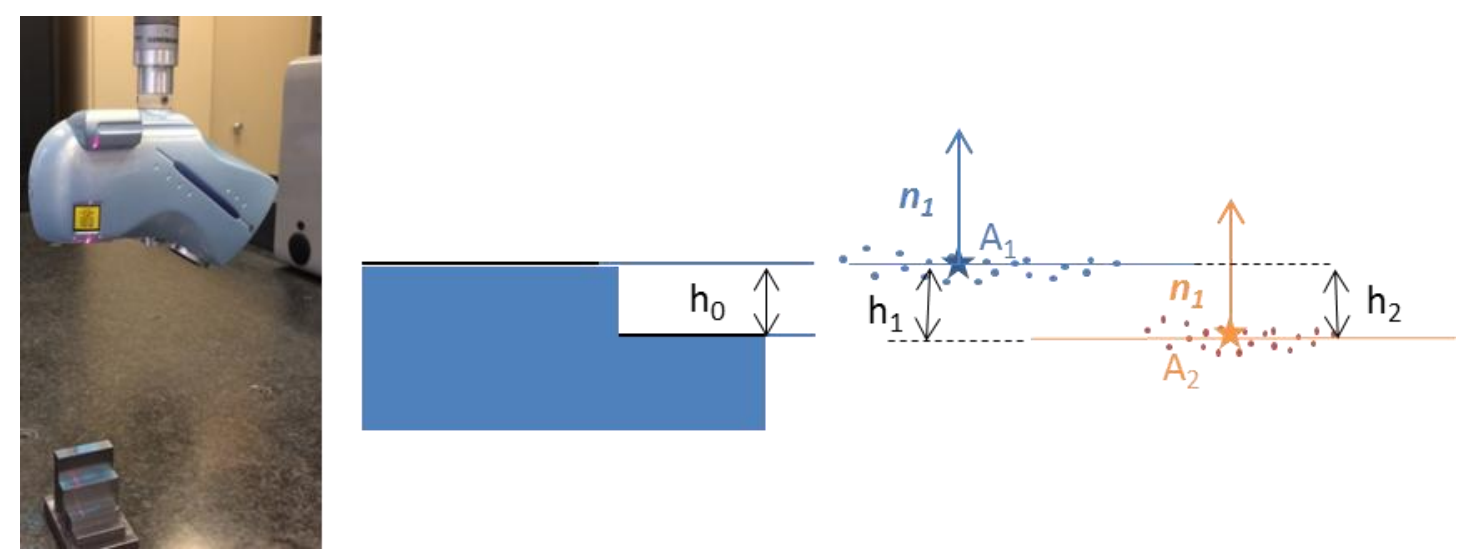

Figure 7.Trueness Evaluation

The trueness is given by eq. 2 in which the calculated height is compared to the theoretical value $h_{0}$.

$T=\frac{1}{2}\left(\boldsymbol{A}_{\mathbf{1}} \boldsymbol{A}_{\mathbf{2}} \cdot \boldsymbol{n}_{\mathbf{1}}+\boldsymbol{A}_{\mathbf{2}} \boldsymbol{A}_{\mathbf{1}} \cdot \boldsymbol{n}_{\mathbf{2}}\right)-h_{0}=\frac{1}{2}\left(h_{1}+h_{2}\right)-h_{0}$

The trueness is evaluated in function ofthe digitizing distance, considering the view angles fixed. The digitizing angles affect the noise that means the dispersion of the points around the ideal element. We make the assumption that the position of the mean associated theoretical element is not affected by the digitizing angle. The distance is adjusted considering the bottom of the step (see figure 8). The evolution of the trueness in function of the digitizing distance is displayed in figure 8 for System 3, considering a 
height step of $10 \mathrm{~mm}$. The figure clearly highlights that the trueness is very good for a large range of distances but varies strongly when D is close to $150 \mathrm{~mm}$. It is important to notice that for this condition, the artefact is at the border of the fov. Therefore, the value reported in the table corresponds to the highest value for a digitizing distance within the range 105-145 mm.In some cases, the trueness is related to the height of the step used.
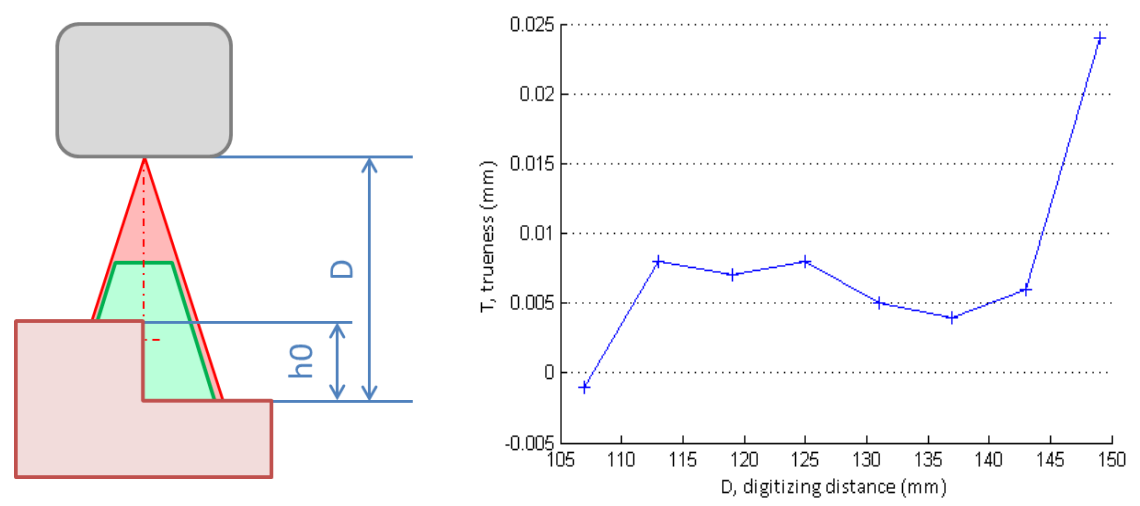

Figure 8.Trueness in function of the digitizing distance

It should be underline that the influence of the device on the quality indicators can be investigated in different ways. For trueness for instance, the artefacts can be of various dimensions in relation with the volume of the working space, and they can be positioned throughout the whole working space. It is also possible to focus on the influence of the joint configuration (for devices such as robots or arms) on the digitizing noise when measuring the reference element.The protocol for quality indicator assessment was subjected to a Matlab development, calledQualiPSO*.

\subsection{Assessment protocol for operational indicators}

Only a few operational indicators are described in the paper, but the approach should obviously be extended to more indicators.

The measured area, $\kappa_{r}$, assesses the digitizing system capacity to measure a given surface in a single view. Indeed, the measured area can give an indication of the percentage of the surface coverage from one single sensor configuration. It is thus possible to obtain the number of relative sensor/object configurations required to reach the completeness. As the curvature of surfaces to be digitized has a major influence on visibility, this indicator is evaluated by measuring a well-known surface: a cylinder with a given radius.

After the digitizing of the surface, a triangular mesh is associated to the points, and the value of the measured area is given by calculating the area of the associated mesh (Figure 4) according to the following equation:

$$
\kappa_{r}=\frac{1}{2} \sum_{i=1}^{n b \text { facets }}\left\|\boldsymbol{M}_{\mathbf{1 i}} \boldsymbol{M}_{\mathbf{2 i}} \wedge \boldsymbol{M}_{\mathbf{1 i}} \boldsymbol{M}_{\mathbf{3 i}}\right\|
$$

A high value of $\kappa_{r}$ means that digitized data give a good representation of the surface.

\footnotetext{
*www.lurpa.ens-cachan.fr
} 

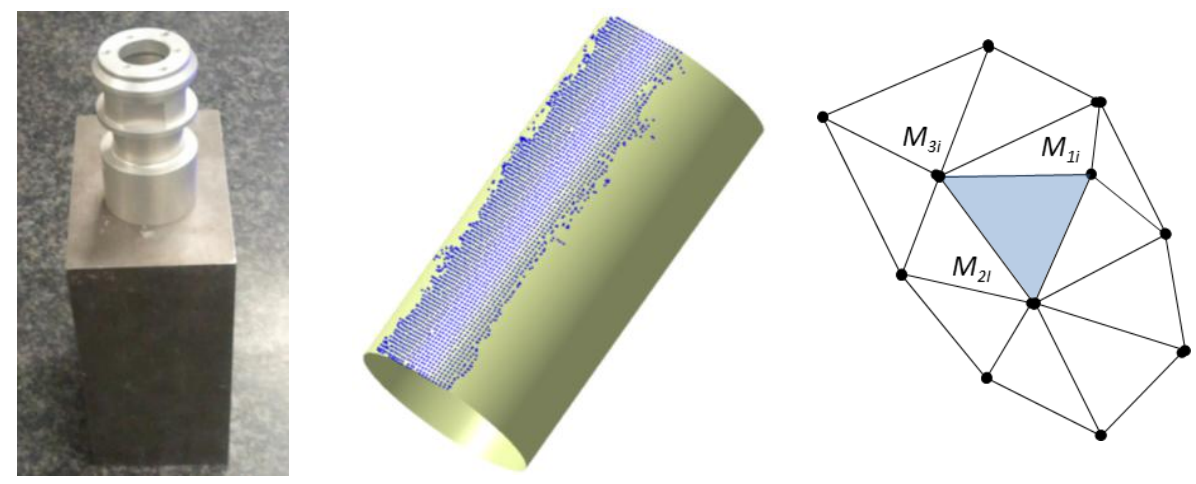

Figure 9.Evaluation of the measured area

The measurement of the cylinder also allows the determination of the angle range $\varphi$. To evaluate $\varphi$, the cylinder is split into sections, and in each section, the angle range $\varphi_{\mathrm{i}}$ is defined by considering the first and the last digitized points as shown in figure 10 . The range angle is defined as the average value of the $\varphi_{i}$ among all the sections.This angle gives an indication on the number of views which would be necessary for a complete digitizing, in relation with the surface curvature.

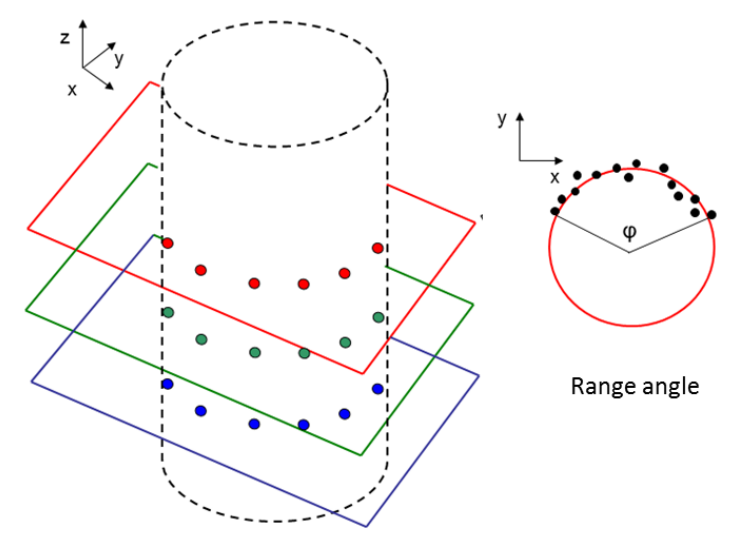

Figure 10. Evaluation of the range angle

Values obtained for both the measured area and the range angle are reported in table 1 for the 3 studied systems. System 2 presents the highest values which is consistent with its pyramidal fov.

The digitizing speedis also a very important indicator in the decision process, but it cannot be considered alone. Indeed, the speed is inevitably associated with a requirement in terms of point density or in terms of data completeness. In this context, the indicator $D_{s}$ is introduced whichaccounts for the digitized area that can be measured per unit of time. $D_{s}$ is computed using the intrinsic factorsgiven by the manufacturers. Whatever the technology, most of the optical sensors are characterized by their field of view, fov.

The size of the fov defines the surface portion which is acquired. For classical laserplane sensor, the fov is planar and can be characterized by its width $W_{f o v}$, whereas it is spatial for most of the structured-light sensors (or sensors by view) and is characterized by its area $A_{f o v}$. As far as laser-plane sensors are concerned, surface acquisition is carried out considering the sweeping of the surface in a direction perpendicular to the laserplane. Let $v_{a}$ be the digitizing speed, generally given by the maximal velocity allowed by 
the displacement system; the digitized area per unit of time $D_{s}$ is given by:

$$
D_{s}=W_{f o v} \cdot v_{a}
$$

In the case of sensors by view, the acquisition is carried out in a single view. Let $t_{a}$ be the associated acquisition time, $D_{s}$ is calculated as follows:

$$
D_{s}=A_{\text {fov }} / t_{a}
$$

Values of $D_{s}$ displayed in table 3 for systems 1 to 3 are thus calculated using eq. 3 or eq. 4 according to the sensor technology.It is important to notice that this indicator only accounts for the area swept by the sensor per unit of time. The density of points belonging to the measured area is not considered.In addition, it could be interesting to define an indicator of digitizing speed directly linked to the point density [7].Note that, only a few operational indicators are considered in the proposed study in order to lighten the paper, but obviously additional indicators could enrich the database.

\subsection{Construction of the qualified database}

Protocols proposed in the previous sections are general and could be applied to a large diversity of digitizing systems [6-7]. Quality and operational indicators obtained are thus incorporated within the qualified database. Quality indicators displayed correspond to the highest values obtained with the protocol. In some cases, a domain of validity can be mentioned. The database will be the support of the best digitizing system selection for a given application (see section 5).

Table 1 Extract of the qualified database

\begin{tabular}{lccc}
\hline Digitizing system & System 1 & System 2 & System 3 \\
\hline Ability indicators & 2 & & 2 \\
On-machine & 1 & 1 & 1 \\
On-line inspection & 1 & 1 & 1 \\
Part flexibility & 2 & 2 & 2 \\
Micro-geometry & & & \\
Quality indicators & 0.009 & 0.002 & 0.017 \\
Noise (mm) & 0.01 & 0.004 & 0.01 \\
Trueness (mm) & 1475 & 1530 & 710 \\
Oper. indicators & 135 & 144 & 77 \\
$\kappa_{r}\left(\mathrm{~mm}^{2}\right)$ & 2500 & 75000 & 30000 \\
$\varphi\left({ }^{\circ}\right)$ & & \\
$D_{s}\left(\mathrm{~mm}^{2} / \mathrm{s}\right)$ & & & \\
\hline
\end{tabular}

As ability indicators are concerned, they account for the system aptitude with regards to a required functionality; that means that the system is able or not able. They are generally built from intrinsic factors. For instance, the ability indicator "microgeometry" directly results from the value of the sensor resolution. By convention, these indicators are inversely proportional to their ability as regards the functionality. Therefore, the indicator takes the value 2 if it is not able and the value 1 if it is able (see Table 1).

To each system $S^{i}$,it is thus possible to associate $n$ indicators which define a vector $\boldsymbol{Q}^{i}=\left[\begin{array}{lll}\boldsymbol{q}_{1}^{i} & \cdots & \boldsymbol{q}_{\boldsymbol{n}}^{\boldsymbol{i}}\end{array}\right]^{t}$ of length $n=n_{a}+n_{q}+n_{o}$, with $n_{a}$ the number of ability indicators, $n_{q}$, the number of quality indicators and $n_{0}$, the number of operational indicators. Each 
indicator has the same importance on the decision process but the associated value is not of the same nature because each category of indicators does not characterize the same thing.The database has been implemented through MySqQL, as displayed in figure 11.

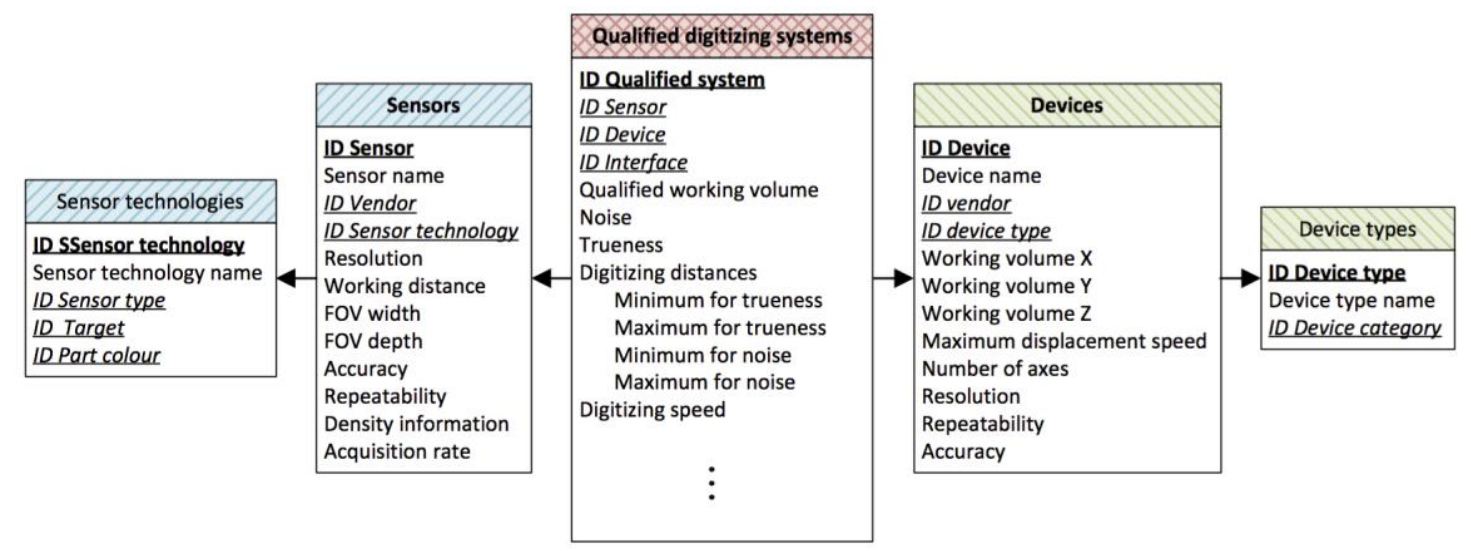

Figure11.Partial representation of the database implementation in MySQL

\section{System selection with regard to applications}

In this section, the database of applications is detailed along with the performance functions allowing the best system selection according to a given application. The approach is finally illustrated thanks to 2 study cases.

\subsection{Database of applications}

The inherent need to an application with regard to 3D digitizing results from the knowledge of an expert. The expert selects its digitizing systems without necessarily justifying his choice or explaining the reasons of his decision. In order to capitalize his knowledge and to make it useable by various users, a database of applications is proposed built from two lists of parameters:

- Aptitude parameters: related to the aptitude of the system to be used for the considered application,

- Performance parameters: related to the relative importance of the qualified indicators with regard to the application.

Aptitude parameters can be seen as decision variables as they tag the system as admissible or not admissible[18]. This involves an ulterior restriction of the 3D digitizing systems to a set of admissible systems. First, it is necessary to define $\boldsymbol{Q}^{\prime \boldsymbol{i}}=\left[\begin{array}{lll}\mathrm{q}_{1}^{\prime \mathrm{i}} & \ldots & \mathrm{q}_{\mathrm{n}}^{\prime \mathrm{i}}\end{array}\right]^{\mathrm{t}}$ from the vector $\boldsymbol{Q}$ as follows:

$$
\begin{cases}q_{k}^{\prime i}=q_{k}^{i} & \text { if } q_{k}^{\prime i} \text { is minimized } \\ q_{k}^{\prime i}=\frac{1}{q_{k}^{i}} & \text { if } q_{k}^{\prime i} \text { is maximized }\end{cases}
$$

For a given application $A^{j}$, aptitude parameters define the aptitudevector $\boldsymbol{A}^{j}=$ $\left[\begin{array}{lll}a_{1}^{j} & \ldots & a_{n}^{j}\end{array}\right]^{t}$ which length is equal to the number of indicators belonging to the system database. The values of $\boldsymbol{A}^{\boldsymbol{j}}$ are given by the expert following a few rules. The idea is that, for the considered application $A^{j}$, a system $S^{i}$ will be considered as admissible with 
regard to a given indicator $k$ if the Heaviside function $H\left(a_{k}^{j}-q_{k}^{\prime i}\right)$ is not zero. For the indicator $k$, the value of $a_{k}^{j}$ corresponds to the admissible threshold of the indicator; the indicator must be lesser than the threshold. Thus, for ability indicators, $a_{k}^{j}=1$ and, when the expert judges thatthe indicator $\mathrm{k}$ has no importance for the application, $a_{k}^{j}=+\infty$.

Performance parameters give the relative importance of the indicators according to the application through a vector of weightings. For a given application $A^{j}$, the expert capitalizes the information that is essential to the selection of the optimal system and defines the performance vector $\boldsymbol{P}^{j}=\left[\begin{array}{lll}\boldsymbol{p}_{1}^{j} & \ldots & \boldsymbol{p}_{n}^{j}\end{array}\right]^{t}$ where $p_{k}^{j}$ are the weightings, with $p_{k}^{j} \geq 0$ and $\sum p_{k}^{j}=1$.

In this first approach, the database of applications is a matrix for which the rows are the applications, and the columns the values of the vectors $A$ and $P$. The application is described relatively simply, and an improvement will be to clearly propose a way to store the context of the application. The values of the vectors $A$ and $P$ are defined by an expert so that his knowledge can be re-usable by other users. This database is more subjective than the database of qualified systems.

Table 2.Database of applications

\begin{tabular}{l||c|c|c|c|c|c|c|c|c}
\hline Applications & $\boldsymbol{a}_{\mathbf{1}}$ & $\boldsymbol{a}_{\mathbf{2}}$ & $\boldsymbol{a}_{\mathbf{3}}$ & $\ldots$ & $\boldsymbol{a}_{\boldsymbol{n}}$ & $\boldsymbol{p}_{\mathbf{1}}$ & $\boldsymbol{p}_{\mathbf{2}}$ & $\ldots$ & $\boldsymbol{p}_{\boldsymbol{n}}$ \\
\hline \hline Application 1 & $a^{1}{ }_{1}$ & $a_{2}^{1}{ }_{2}$ & $a^{1}{ }_{3}$ & $\ldots$ & $a_{n}^{1}$ & $p_{1}^{1}$ & $p_{2}{ }_{2}$ & & $p_{n}^{1}$ \\
Application 2 & $a^{2}{ }_{1}$ & $a_{2}^{2}$ & $a_{3}^{2}$ & $\ldots$ & $a_{n}^{2}$ & $p_{1}^{2}$ & $p_{2}^{2}$ & & $p_{n}^{2}$ \\
$\ldots . .$. & & & & & & & & & \\
\hline
\end{tabular}

\subsection{Decision process}

Once the two databases are filled, the first one thanks to the assessment protocol and the second one via the expert knowledge, the selection of the best system $S^{i}$ for an application $\mathrm{A}^{\mathrm{j}}$ can be carried out through a decision process. This process consists of 2 main stages: the restriction of the initial set of systems to a set of admissible systems, and the selection of the optimal one among admissible systems.

The stage of restriction is performed thanks to a function $f_{1}$ built from aptitude parameters:

$$
f_{1}(i, j)=\prod_{k=1}^{n} H\left(a_{k}^{j}-q_{k}^{\prime i}\right)
$$

If $f_{1}(i, j)$ is non null, then the system $S^{i}$ is tagged as admissible with regard to the application $\mathrm{A}^{\mathrm{j}}$. A subset of admissible system $S_{a}(j)$ is thus defined :

$$
S_{a}(j)=\left\{S^{i} \in D B_{\text {system }}, f_{1}(i, j)>0\right\}
$$

The second stage is the optimization of a performance function $f_{2}$ in order to define the best system. The best systembelongs to $S_{a}(j)$ and minimizes the cost function:

$$
f_{2}(i, j)=\left(\boldsymbol{P}^{j}\right)^{t} \cdot \Delta \cdot \boldsymbol{Q}^{\prime \boldsymbol{i}}
$$


Where $\Delta$ is a diagonal matrix defined by $\Delta(\mathrm{k}, \mathrm{k})=\left(\max _{i}\left(\mathrm{q}_{\mathrm{k}}^{\prime \mathrm{i}}\right)\right)^{-1}$ used to normalize aptitude indicators.

\section{Study cases}

First, the approach is applied to crankshaft digitizing. Some new innovations in automotive industries tend to propose methods for crankshaft balancing based on the acquisition of the part surface geometry. Such methods should lead to a more flexible and far less expensive balancing. Previous works have showed the relevance of part balancing by geometry measurement, and have also defined the digitizing requirements in terms of quality to obtain the values of the unbalance vectors consistent with the industrial requirements |6]. The maximum error tolerated for respecting balancingrequirements is $5 \%$. This involves thatthe admissible noise $\delta_{a d m}=0.083 \mathrm{~mm}$ and the admissible trueness is equal to $T_{a d m}=0.01 \mathrm{~mm}$ for a step height of $1 \mathrm{~mm}$. On the other hand,crankshafts are rigid parts including some complexity for the sensor accessibility which imposes the largest field of view in order to limit sensor repositioning. Finally, as time cycle is important for on-line process, as it is the case for crankshaft balancing, digitizing speed is the most important factorfor digitizing system selection.

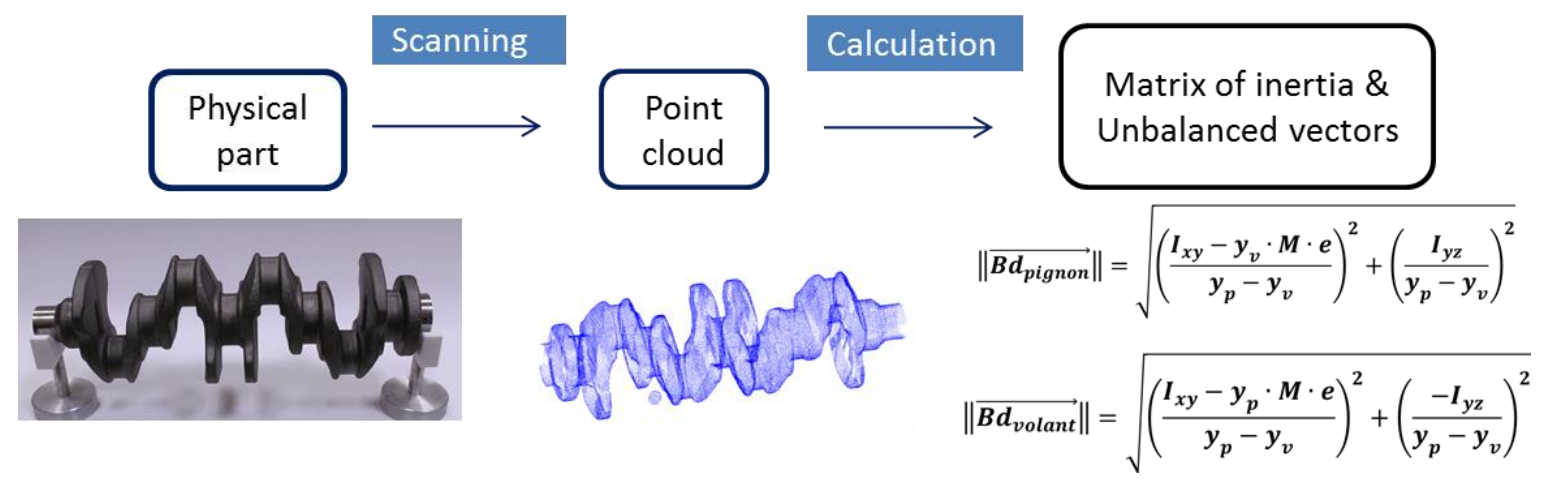

Figure12. Crankshaft balancing by digitizing of the external geometry

These requirements lead to the aptitude and to the performance vectors:

- $\quad$ Aptitude: $\boldsymbol{A}^{1}=[+\infty+\infty+\infty+\infty 0.0830 .01+\infty+\infty+\infty]^{\mathrm{t}}$

- $\quad$ Performance: $\boldsymbol{P}^{\mathbf{1}}=\left[\begin{array}{lllllllll}0 & 0 & 0 & 0 & 0 & 0 & 0 & 0 & 1\end{array}\right]^{\boldsymbol{t}}$

Therefore, according to eq. (5) and considering the systems of table 1 , the function $f_{1}$ is non null for all the systems. The second step is thus to evaluate the performance function $f_{2}$, for the admissible systems. Consideringvalues of table $1 f_{2}(1,1)=1, f_{2}(2,1)=1 / 30$ and $f_{1}(3,1)=1 / 12$, System 2 is thus the best system for the application of crankshaft balancing.

The second application focuses on on-line inspection of rough-forged partsusing a digitizing system. This corresponds to the assessment of the rough part at the beginning of the crankshaft balancing line. Concerning ability, the "on-line inspection" indicator is essential. For quality indicators, the quality of the acquired point cloud must allow the verification of geometrical specifications. It is classically admitted that a measuring system is said admissible with regard to the specification verification if its uncertainty is less than IT/8, where IT is the tolerance interval associated with the specification. In this 
application, the aim is to check that the general form deviation is less than $0.8 \mathrm{~mm}$. The associated admissible uncertainty is thus $u=0.1 \mathrm{~mm}$ which leads to the value of the admissible noise $\delta_{a d m}=0.017 \mathrm{~mm}$.As the measurement is performed on-line, the measured areaandthe digitizing speed are essential in the optimization process to limit acquisition time. The maximal weighting is given to the measured area in order to limit registration errors. Thus:

- Aptitude: $\boldsymbol{A}^{2}=[+\infty 0+\infty+\infty 0.017+\infty+\infty+\infty+\infty]^{\mathrm{t}}$

- $\quad$ Performance: $\boldsymbol{P}^{2}=\left[\begin{array}{lllllllll}0 & 0 & 0 & 0 & 0.25 & 0 & 0.5 & 0 & 0.25\end{array}\right]^{\mathrm{t}}$

Concerning aptitude, the function $f_{1}$ is non null for all the 3 systems. Finally, this leads to $f_{2}(1,2)=0.640, f_{2}(2,2)=0.274$ and $f_{2}(3,2)=0.771$. System 2 is thus the best-system for evaluating the form deviation of rough crankshafts thanks to on-line digitizing.

\subsection{Discussion}

Once the digitizing system has been chosen, it is possible to generate the optimal digitizing strategy. In our study, System 2 is a structured light system set on a tripod, and the digitizing points of view are calculated in function of the fov size with the aim of defining a minimal set of viewpoints[19]. The 50 calculated viewpoints allow the crankshaft digitizing in about 16 minutes, considering a manual repositioning. As it can be seen in figure 13, completeness is almost reached. Calculation of the unbalance vectors is performed with an error of less than 5\%, relatively to classical mechanical balancing methods. This result was expected, and confirms the efficiency of the proposed method to select the best digitizing system. However, acquisition time is not optimal due to manual repositioning. A possible optimization could be the automation of the process using a robot, as proposed in figure 12 (right).

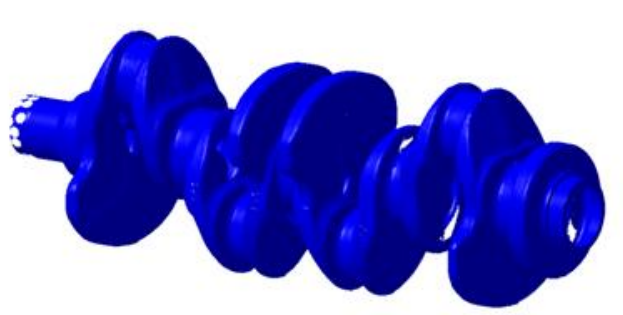

Crankshaft point cloud

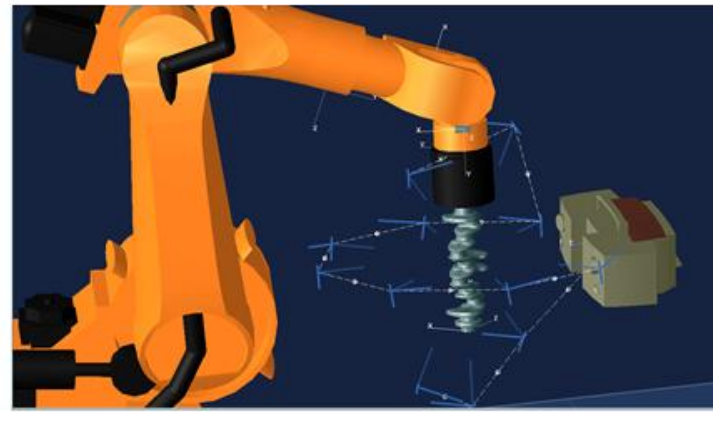

Figure 13.Crankshaft digitizing using system 2

\section{Conclusion}

In this paper, a non-subjective method was proposed to select the most suitable digitizing system with regard to a given application. For this purpose, an efficient knowledge database of qualified digitizing systems has been established according to performance indicators. The proposed indicators are classified into three main categories: ability, quality and operational indicators. Quality and operational indicators are obtained thanks to assessment protocols. These generic protocols are voluntarily simple with the aim of being applicable to a large diversity of digitizing systems. On the other hand, a database of applications which capitalizesexperts' knowledgeis built 
considering two types of parameters: aptitude and performance parameters. To support the optimal digitizing system selection according to a given application,the two databasesare coupled with a decision process based on the minimization of a cost function.As an illustration, the method has been applied to select the best digitizing system for two different applications.

Future works will focus on the extension of both the databases of systems and of applications. It could be interesting to enrich the qualified database by proposing additional operational indicators. In particular, integrating indicators linked with the part geometry complexity seems essential to enlarge the number of applications.Another way of improvement concerns a method to capitalize and/or reuse expert's knowledge. It would be necessary to propose a method to formalize the variables defining an application and its context, so that the $\mathrm{A}$ and $\mathrm{P}$ vectors could be defined automatically using case-based reasoning for instance or other techniques of artificial intelligence.Our final objective is to give public access to online qualified and application databases.

\section{References}

[1] Bernard, A., Laroche, F., Ammar-Khodja, S., Perry, N, Impact of New 3D Numerical Devices and Environments on Redesign and Valorisation of Mechanical Systems, Annals of the CIRP, 2007, 5/1:143-148

[2] Savio,E., De Chiffre, L., Schmidtt, R., Metrology for shaped parts, Annals of the CIRP, 2007,56/2:810-935

[3] Loriot B., Automatisation de l'acquisition et des post-traitements en numérisation 3D, Thèse de Doctorat de l'Université de Bourgogne, France, 2009

[4]Barbero, B. R., Ureta, E. S., Comparative Study of different digitization techniques and their accuracy, Computer-Aided Design, 2011, 43/2:188-206

[5] Zhao, H., Kruth, JP., Van Gestel, N., Boeckmans, B., Bleys, P.,Automated dimensional inspection planning using the combination of laser scanner and tactile probe, Measurement,2012,45:1057-1066

|6] Zuquete-Guarato, A., Mehdi-Souzani, C., Quinsat, Y., Lartigue, C., Sabri, L., Towards a new concept of on-line crankshaft balancing by contact less measurement: process for selecting the best digitizing system,CDrom paper $n^{\circ}$ 82166, ESDA 2012, July 2-4 2012, Nantes, France

[7] Audfray, N., Mehdi-Souzani , C., Lartigue, C., Assistance to automatic digitizing system selection for 3D part inspection, ESDA 2012, CDrom paper $n^{\circ} 82319$, July 2-4 2012, Nantes, France

[8] Singh, R., Gernaey, K.V., Gani, R., An ontological knowledge-based system for the selection of process monitoring and analysis tools, Computer and Chemical Engineering, 2010,34:1137-1154

[9] Chandrasegaran, S. K., Ramani, H., Sriman, R.D., Horvath, I., Bernard, A., Harik, R.F., Gao, W., The evolution, challenges and future of knowledge representation in product design systems, Computer-Aided Design, 2013, 45:204/228

[10] Wu, J., Houssin, R., Bernard, A., Caillaud, E., Systemic modeling of knowledge for innovation and design, CIRP journal of Manufacturing Science and technology, 2013,6:112 
[11] Lartigue, C., Contri, A., Bourdet, P., Digitised point quality in relation with point exploitation, Measurement, 2002, 32/3:193-203

[12] Mahmud, M. , Joannic, D., Roy, M. , Isheil, A. ,Fontaine, J.-F, 3D part inspection path planning of a laser scanner with control on the uncertainty, Computer-Aided Design,2011, 43/4:345-355

[13] Prieto, F., Redarce, T., Lepage, R., Boulanger, P., An automated inspection system, International Journal ofAdvanced Manufacturing Technology, 2002,19: 917-25.

[14] VanGestel, N., Cuypers, S., Bleys, P., Kruth, J-P., A performanceevaluationtestforlaserlinescannersonCMMs, Optics and Lasers in Engineering, 2009, 47, 336-342

[15] Mehdi-Souzani, C., Lartigue, C., Contact less laser-plane sensor assessment: toward a quality measurement,Proceedings of IDMME-Virtual Concept 2008,Beijing (China),CDRom paper $\mathrm{N}^{\circ}$ 41,9p.,October 2008

[16] Feng, H-Y., Liu,Y.,Xi, F., 2001, Analysis of digitizing errors of alaser scanning system. Precision Engineering, 25(3):185 _ 191

[17] IS05725-1, 1994, Accuracy (trueness and precision) of measurement methods and results - Part 1: General principles and definitions

[18] Kashkoush, M., ElMaraghy, H., Knowledge-based model for constructing master assembly sequence, Journal of Manufacturing Systems, 2015, 34: 43-52

[19] Lartigue, C., Quinsat, Y., Mehdi-Souzani, C., Zuquete-Guarato, A., Tabibian, S., Voxelbased path planning for 3D scanning of mechanical parts, Computer-Aided Design and Applications, 2014, 11/2:220-227 